\title{
Bioinformatics approaches for identifying new therapeutic bioactive peptides in food
}

\author{
Nora Khaldi ${ }^{1,2}$ \\ ${ }^{\mathbf{1}}$ School of Medicine and Medical Science, University College Dublin, Ireland; ${ }^{2}$ Food \\ Science and Technology, University College Davis, Davis, CA, USA
}

Corresponding author: Dr. Nora Khaldi, PhD, Office 2G3, UCD Complex and Adaptive Systems Laboratory (CASL), University College Dublin, Belfield, Dublin 4, Ireland

Submission date: August 03, 2012, Acceptance date: October 12, 2012; Publication date: October 15, 2012

\begin{abstract}
:
The traditional methods for mining foods for bioactive peptides are tedious and long. Similar to the drug industry, the length of time to identify and deliver a commercial health ingredient that reduces disease symptoms can take anything between 5 to 10 years. Reducing this time and effort is crucial in order to create new commercially viable products with clear and important health benefits. In the past few years, bioinformatics, the science that brings together fast computational biology, and efficient genome mining, is appearing as the long awaited solution to this problem. By quickly mining food genomes for characteristics of certain food therapeutic ingredients, researchers can potentially find new ones in a matter of a few weeks. Yet, surprisingly, very little success has been achieved so far using bioinformatics in mining for food bioactives.

The absence of food specific bioinformatic mining tools, the slow integration of both experimental mining and bioinformatics, and the important difference between different experimental platforms are some of the reasons for the slow progress of bioinformatics in the field of functional food and more specifically in bioactive peptide discovery.

In this paper I discuss some methods that could be easily translated, using a rational peptide bioinformatics design, to food bioactive peptide mining. I highlight the need for an integrated food peptide database. I also discuss how to better integrate experimental work with bioinformatics in order to improve the mining of food for bioactive peptides, therefore achieving a higher success rates.
\end{abstract}

Keywords: bioactive peptides, bioinformatics, mining food, therapeutic properties, food proteins, functional food.

\section{INTRODUCTION:}

Food fermentation is used as a means to create food products with different or better taste, different functional properties, or with therapeutic qualities that otherwise are absent in the initial food. Human consumption of fermented food is an ancient practice however it is 
estimated that it began with the creation of cheese over 8,000 years ago. One of the major changes that fermentation can bring to food is hydrolyzing the food-proteins into smaller fragments, known as peptides. Some of these peptides have been shown to have a relevant impact on human health. For example bioactive peptides from various food sources have been shown to lower blood pressure, broccoli, egg, milk, meat, wheat, soy, and fish . Some peptides found in milk are anti-microbial, a similar functionality is also found in egg .

I have witnessed in the past decade a shift in public awareness towards how certain elements contained in food can enhance ones health and therefore reduce the need for artificial drugs. This has in turn alerted food companies to an attractive new market segment. This has meant that important research has been dedicated by the food industry to mining food with the aim of developing functional foods, such as products containing bioactive peptides.

The traditional way of finding these bioactive peptides is a very long and a tedious process. Very often this process includes random digestion of food followed by experimental testing of hundreds of versions of a hydrolysate. But the most tedious part is then to determine the bioactive set of peptides that are presenting the activity within the active hydrolysates. The time and cost associated with this more traditional and purely experimental approach results in a small number of commercial products with bioactive ingredients. Indeed, less than 20 food-peptide based products are commercially available (see for a detailed table about commercially available peptides), some of these include Calpis AMEEL S (Japan) and Calpico with a hypertensive effect. Davisco also created BioPURE-GMP, which is a peptide mixture with anticariogenic, antimicrobial and antithrombotic health benefits. Another example is the Glutamin peptide by DMV, which carries an immunomodulatory activity.

Because of the above, more time, money and effort is being spent trying to detect novel bioactive elements in food. This shift has been accompanied with an expansion of nutraceutical and functional food research. The development of high throughput methods and techniques such as mass-spectrometry is resulting in tremendous amounts of data. Massspectrometry has been used for example to investigate the types of peptides found in a hydrolysate. As a result of the slow experimental route, bioinformatics was introduced as the solution to quickly mine for bioactive peptides in food. Indeed this seems like an obvious idea given the dramatic scientific leap that bioinformatics allowed the drug industry to take over the last two decades.

However, the emergence of bioinformatics in food research has not been straightforward and to date very few results have been delivered. There are many reasons to explain the slow success rate of bioinformatics in food bioactive peptide mining, but three major ones are worth mentioning. First, it is not straightforward to translate the tools developed in bioinformatics in the drugs industry to mining for bioactive peptides in food. The drug industry has very different pressure to that of the food industry. The role of pharmacy is to find new drugs whatever the cost and time. In contrast it is only recently that the food industry is realizing that functional bioactive food is the food of the future and may be worth the investment to deliver products for this market. However this is still not a necessity, but rather an adaptation to a growing market need and mainly a future requirement. So while drug companies have always walked in parallel with state of the art technology and science, this is not the case for the food industry that needs to not only catch up in food mining but in the related general science in this area. Secondly, most of the bioinformatic 
tools used in drug discovery is based on drug docking of structurally defined molecules, while, most of the bioactive peptides are short and thus hard to identify a structure that they will adopt outside the full protein. This shows that new bioinformatics tools need to be developed to meet the special requirements of mining for food bioactives as opposed to just translating the drug mining tools. Finally, there is very slow integration of both, experimental, and the in-silico food mining research. While there is pressure in the drug industry to adopt consensus methods and have worldwide databases, it is not the case for the food industry, where many of the methods are very different from lab to lab, and thus hard to find a generic consensus or structure. This is shown by the absence of databases for bioactive peptides widely used by the food industry and academics.

The purpose of this review is to provide a possible set of bioinformatic methods that could be used to aid food research and industry with bioactive peptide mining. Here again, because of the lack of literature on the trial and error of these methods it is hard to quantify their success rate. But I do explain how some would have been successful in detecting known bioactive peptides. The focus will also be to highlight the need for a worldwide database that food research can update and consult on a daily basis regarding food bioactive peptides. In particular I will discuss how computational biology can be integrated with experimental methods in the most efficient way to increase the yield of new bioactive peptides found in food.

\section{Techniques used to find bioactive peptides:}

I illustrate here different methods some of which have been successfully used to find new bioactive peptides. Most of these methods are bioinformatic based, or could be easily translated into systematic bioinformatic approach. Although most of these approaches were not used on food, they have great potential in finding new bioactive peptides in food.

Method 1: Evolutionary conservation may play a key role in identifying new food bioactive peptides for some diseases

Edwards and coauthors were interested in finding new bioactive peptides that showed a significant effect on human platelet function. This work represents a good example whereby the initial mining is performed using bioinformatics, which allowed the team to focus on a small number of candidates that are most likely carrying the activity of interest. The initial set of platelet expressed candidate proteins they had was 2900. It would have been very costly and lengthy to test regions of all 2900 proteins. Besides, a random selection of some proteins, followed by a random selection of peptides, is very unlikely to yield results. Instead, the authors applied a rational peptide design approach specifically targeted at juxtamembrane regions that were predicted to have signaling activity and could be modulators of platelet function. To narrow down the space of search to candidates most likely carrying the desired functionally the authors used bioinformatics to identify 47 candidates highly expressed transmembrane proteins with signatures of functionally specific residues in cytoplasmic tails or loops. They carried out this using an evolutionary approach whereby the chosen peptides span residues that are strongly similar (conserved) to those in the corresponding proteins in other species (orthologs), but that differ from those in related human proteins (paralogs). An example is illustrated in Figure 1-A using a milk protein example. The authors reasoned that because the functionality of platelet aggregation is crucial to the cell, the regions that support and perform this function should be conserved but will not be present 
in a related protein that has a different function in that region. The peptides also had to lie within 30 amino acids of the membrane and meet certain synthesis and solubility criteria .

This computational approach allowed the experimental biologist to test candidates that most likely carry this functionality rather than random candidates. The results showed that 26 peptides have significant effect on platelet function. This significant finding would not have been possible without the initial bioinformatic screening.

Method 2: Amino-acid composition and preference can define some diseases allowing for the discovery of new food bioactive peptides

A.

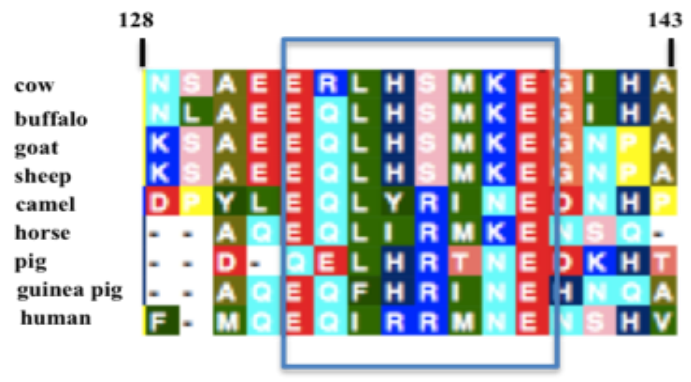

B.

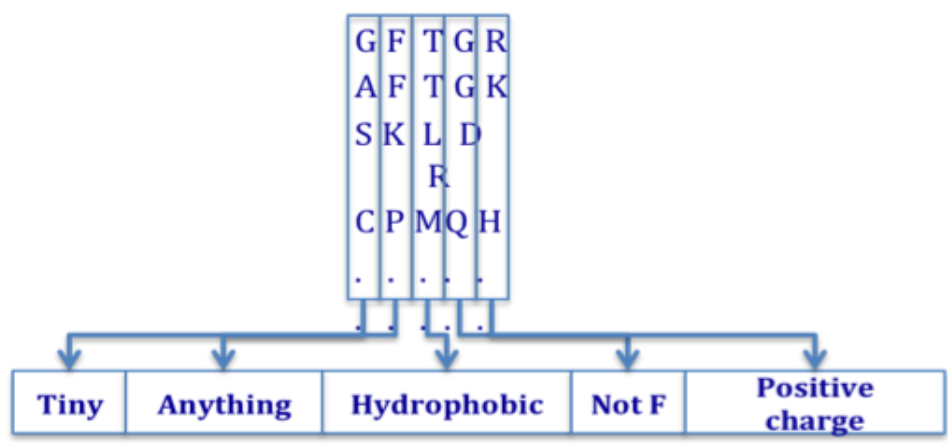

C.

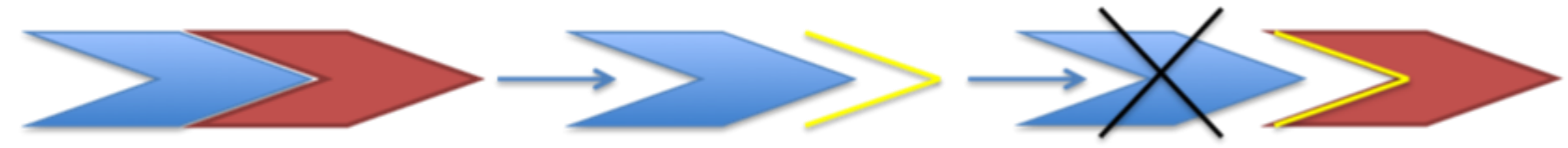

Figure 1. Representation of three different methods used to find new bioactive peptides. A. Alignment of the region 128-143 of bovine $\alpha$-S1-casein between related species. The blue box surrounds the region that is conserved relatively to the surrounding region in the protein. B. Mimicking a region of interaction between two proteins, the new peptides can inhibit the interaction of both proteins. C. Example of amino acid preference underlining the functionality of a set of peptides shown to reduce a given disease. For the figure purpose I chose to only show 4 peptides, numerous other peptides are represented by dots.

Often bioactive peptides perform a given function because of their structure and amino acid composition. Figure 1-B is an illustration of an example where patterns of amino acids are established based on amino acid preferences at certain positions for a number of peptides all carrying a similar functionality. For example Jianping $\mathrm{Wu}$ et coauthors carried out an interesting study to examine the quantitative structure-activity relationships of angiotensin Iconverting enzyme (ACE) inhibitory peptides. These peptides have a blood pressure lowering effect, and are thus interesting for the cardiovascular functional food community. Using a computational approach partial least squares regression they found that di-peptides carrying an ACE inhibitory function preferred residues with bulky side chains as well as hydrophobic 
side chains. For tri-peptides, the most favorable residues for the carboxyl terminus were aromatic amino acids, positively charged amino acids were preferred for the middle position, and hydrophobic amino acids were preferred for the amino terminus. These patterns allowed the authors to mine for peptides that carry similar structures. They mined pea, bovine milk, and soybean proteins and predicted 7 new biaoctive peptides (3 di-peptides, and 4 trypeptides) that are likely carrying an ACE-inhibition effect.

Method 3: Mimicking a Binding Partner to discover new bioactive peptides:

In this paragraph I describe an interesting approach that has been very effective in the drug industry. This mimicking approach, illustrated in Figure 1-C, uses a reasoning that can easily be translated into a bioinformatics approach to mine for bioactive peptides in food.

Protein-protein interactions are difficult to inhibit. Thus it is not evident to expect that a peptide may inhibit a Protein-protein interaction by mimicking a region of the protein where the interaction takes place. Indeed peptides can be small and one may expect low structure conservation relative to the protein. Interestingly and unexpectedly, a study carried out on protein kinase $\mathrm{C}$ ( $\mathrm{PKC}$ ) has demonstrated that competitive inhibition of this protein by a peptide that mimics the interaction region of this protein is possible.

PKC are a family of serine/threonine kinases, which are involved in many signaling events. These enzymes are associated with many diseases including cancer, diabetes, heart attack, stroke, and heart failure. Because of this association great attention has been made to generating PKC isoenzyme-selective inhibitors that target the active enzyme. This approach has been - to say the least- challenging.

This all started with the observations made by Alistair Aitken that 14-3-3, a protein he found to inhibit PKC, and annexin I, a protein found by Mochly-Rosen and co-workers to bind $\beta \mathrm{PKC}$, share a 12-amino-acid sequence of homology triggered an interesting idea. Mochly-Rosen and co-workers, reasoned that because these two unrelated proteins share this region, and that both bind to $\mathrm{PKC}$, this region may mediate the binding of both proteins to PKC.

Mochly-Rosen and co-workers, decided to investigate the action of this peptide (consequently named peptide I) on PKC interaction with RACK and annexin I. The results showed that consequently peptide I prevented the access of the active enzyme to its substrates, leading to inhibition of the downstream physiological responses (the anchoring of activated PKC to RACK is inhibited).

This approach has since been used in many other studies. From our perspective it is easy to see how this approach could be computationally applied to thousands of interacting proteins to yield possible common regions responsible for these interactions. This competitive inhibition of PKC by a region that mimics its interaction with other proteins is potentially a good method to identify new functional food peptides. The idea is that unrelated protein interacting with the same protein may share a common region allowing this binding to occur. In other words, a pathway may be promoted or inhibited via these mimicking peptides.

Another similar example of the presence of a similar sequence in unrelated proteins is the presence of KEN-box motifs in unrelated proteins. Indeed these motifs are used by the Anaphase-Promoting Complex (APC/C), which is a ubiquitin ligase complex that plays a major role in regulation of signal transduction, to mark cell cycle proteins for degradation by the $26 \mathrm{~S}$ proteasome. Using a computational approach Sushama Michael and co-authors found many new instances of KEN motifs in many proteins . The authors used protein intrinsic 
disorder and evolutionary conservation to separate between possibly functional and nonfunctional KEN motifs. Indeed intrinsic protein disorder has been shown to be rich in motifs and evolves similarly between proteins of the same complex.

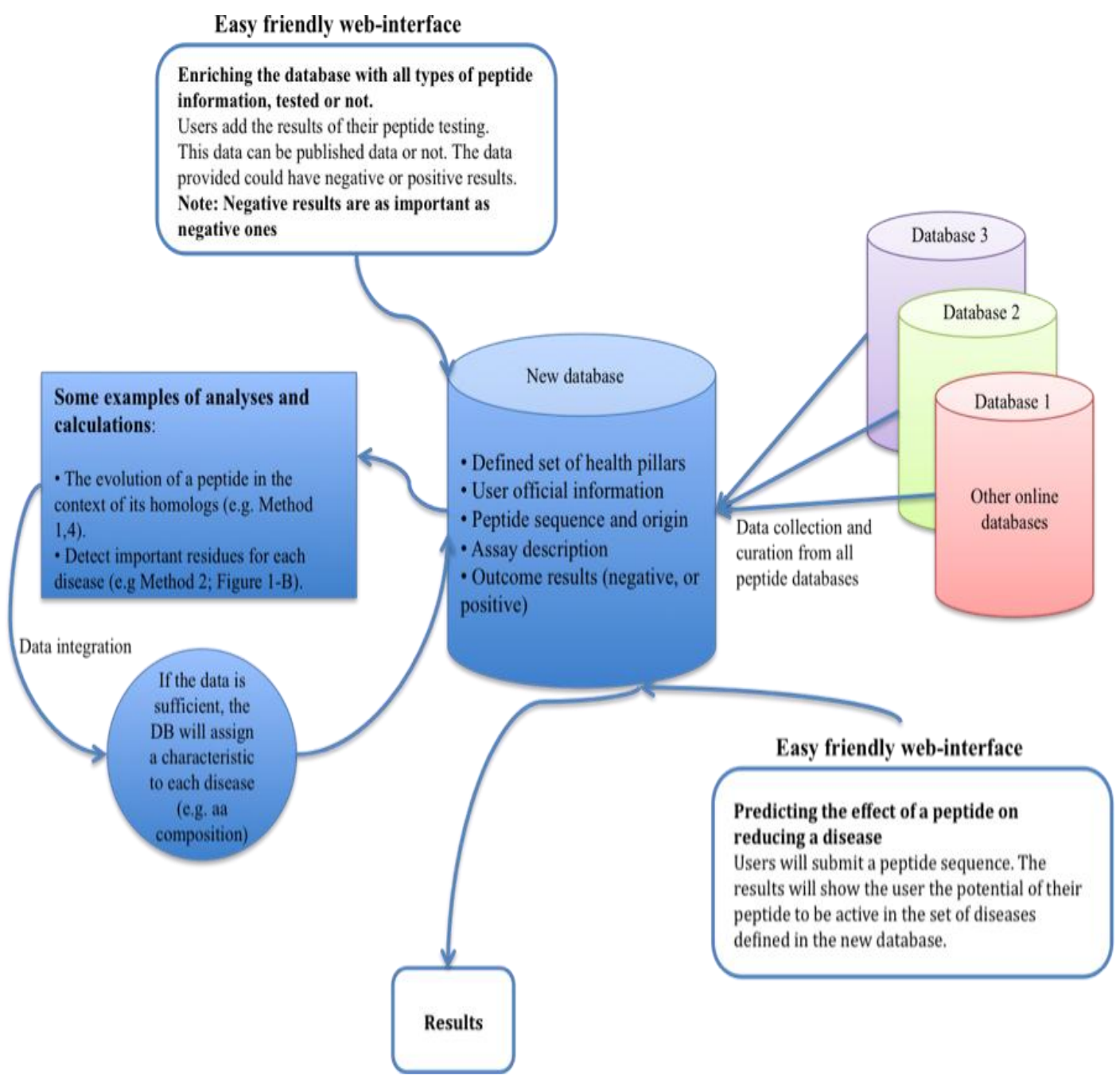

Figure 2. Schematic representation of a centralized database for functional food peptides. A user can either add information into the database by giving the activity they investigated, the peptides they tested, the assay they used, and the positive or negative results they found. DB stands for database, and aa for amino acid.

Method 4: Positive selection may play a key role in identifying new bioactive peptides in food proteins:

When I and Prof Denis Shields investigated the significant shift in charge and isoelectric point of milk proteins between different species we realized that an important contributor to these shifts are the significant differences between amino acids composition at certain positions in different mammalian milk proteins. Many of these residues we predicted to be under positive selection. But a major side observation we had made from these results is the overlap between bioactive peptides, found in bovine milk $\kappa$-casein, and residues that are under positive Darwinian selection (this is illustrated in Figure 4 of). Most of the known literature peptides in $\kappa$-casein seem to either overlap over these residues or be adjacent to them in the parent sequence. One of the results of this study is that positive selection seems to be an indicator for the presence of a bioactive peptide. In other words, residues in bioactive 
peptides or surrounding them seem to be under fast sequence evolution, perhaps the consequence of a species adaptation to varying needs in different species. It would be interesting in further studies to see if this observation extends to other known bioactive peptides.

The above seems to contradict the evolutionary conservation method used by Edwards and coworkers to define novel platelet aggregation peptides. It is expected that some functions are widely needed in many species and are expected to be conserved, while other are more species specific, for example anti-opioid function seems to be an activity that is not conserved (casoxin-A; ), and thus species specific. Thus, both methods are not a contradiction per se but the result of the different functionalities carried by a bioactive peptide that may need to be conserved or not.

A possible way for finding bioactives in a protein sequence using this method is to investigate the residues under positive selection and test the peptide that contain these selected residues. Delineating the peptide will be somewhat challenging and would possibly require a few trials and errors.

\section{Possible success using the methods above:}

The mechanisms of action of many of the commercial or literature known food bioactive peptides remain unknown. Thus it is hard to measure the success rate of approach 1 and 3 on re-discovering these known peptides, as both these methods require a particular knowledge of what pathways or interactions one wants to target. However using method 2, which only depends on the sequence, would have discovered the tri-peptide "IKW" an ACE-inhibitory peptide. Furthermore using method 4, which also depends on the protein sequence would have allowed us to discover the Opioid antagonist Casoxin $\mathrm{A}$ and $\mathrm{C}$ in $\kappa$-casein, and at least highlight another Opioid antagonist in $\kappa$-casein namely Casoxin B , and the antithrombotic peptide Casoplatelin also found in $\kappa$-casein.

Method 1 yielded positive results most likely because the assayed effect -a platelet response- is an inherent part of the native protein function. The use of this method without some prior knowledge of the disease targets or protein interactions required to yield a positive effect on the disease under consideration will be of ineffective. Once this bigger picture is set for a given species such as human, mining for peptides in the homologous proteins in plants of their counter parts in human becomes feasible. And method 1 would potentially lead to significant results

Similarly to method 1 , the use of method 3 will require prior knowledge of what interaction we would like to inhibit. Once this knowledge is established the identification of peptides in food proteins that would mimic the interacting protein becomes possible.

\section{Other existing methods to predict functional regions in a protein sequence.}

There are some other methods to predict functional regions in protein sequences, these include searches for Molecular Recognition Features (MoRFs) and various short linear motif (SLiMs). But from my own experience these predictors have failed to predict novel, functional, and commercially viable bioactive peptides. A possible explanation for this is that SLiMs for example are usually found in disordered regions of a protein. It has been shown for example that differences between disordered regions in duplicates can cause functional divergence given that some will acquire new motifs while others do not. Protein disordered 
regions happen to also be post-translationally modified regions, phosphorylated and glycolsylated. This fact makes it very hard for enzymes to reach and cleave potential peptides in these regions. So although these disordered sequences may have peptides, they will be impossible to reach easily without heavily modifying or breaking the protein, something the food industry will not be too keen on, for many reasons one of which is taste. Further research in this area will allow a better understanding of the relationship -if any- between MoRFs and/or SLiMs and the bioactive food peptides.

\section{A worldwide database for bioactive peptides: Includes information about peptides that presented an activity and ones that did not present any activity:}

In order to systematically develop bioinformatic methods to analyze, predict bioactive peptides, and aid researchers in discovering new ones it is important to create a central database (Figure 2). This database will centralize all available information about peptides that have been tested on an assay. The type of assay should be predefined in the database, where the user will only need to select the assay they have used. This is to minimize results that are not conformational to known and widely accepted assays. What is crucial for any predictions is the use of published and unpublished data. Many peptides have been tested but yielded negative results in some assays. This unwanted data is currently an unused mine of information. Indeed, these negative results are as crucial as is the information about the peptides that yield positive results. One may not observe any commonalities between peptides that have shown a positive action on an assay in relation to a disease, but contrasting this information with peptides that have shown negative effects in the same disease related assays may lead to discovering the key characteristics needed for the functionality of these peptides.

This central database will not only contain food related bioactive peptides, but will integrate with all peptide databases resources to enrich the set of peptides for each disease thus increasing the prediction power for novel bioactive peptide (Figure 2).

As mentioned above, there seems to be no clear-cut and general method for discovering novel bioactive peptides. Some methods may be good at predicting bioactive peptides for a certain disease but do not work on another. For this reason, it is important to implement all these different methods automatically on each group of peptides (Figure 2; a group of peptides is defined as a set of peptides that share a common characteristics such as they all lower cholesterol). The data that is constantly updated in the central database will be passed through different bioinformatic methods, such as the ones described above. For example, an amino acid preference matrix will be constructed for each disease. An evolutionary characterization of each peptide, whereby the closest homologs of the proteins that contains these peptides are aligned to determine signals of positive selection, which seems to be an indicator for the presence of a bioactive peptide (Figure 1-b).

\section{The bigger picture: effectively integrating bioinformatics and experimental work to mine for new food bioactive peptides.}

Here I recommend a process for effectively discovering novel bioactive peptides. The aim of these approaches is to accelerate the tie between finding an active hydrolysate and discovering the peptides responsible for this activity. This process will integrate three different approaches as outlined in Figure 3 and discussed in more detail below.

The most effective and commonly used method for identifying new bioactive peptides 
is the generation of digested food or hydrolysates (Figure 3, top panel). Corn for example will undergo digestion with a combination of different enzymes, or different bacterial organisms under different conditions ( $\mathrm{pH}$, temperature, time in contact with enzyme). This method is purely experimental and will produce hundreds of different hydrolysates that are then experimentally tested in different assays representing different diseases.

The aim of any organization conducting this study is the commercialization of the positive results. A positive hydrolysate that actively lowers a disease can either be commercialized as is, in which case the claims on this product are limited. Or it can be further mined to find the bioactive element(s) and thus improve the claim. Using a computational approach at this point is crucial in handling these amounts of data and predicting what fragments are the most likely bioactive in each positive hydrolysate. Indeed a purely experimental approach would result in years of trial and error, a luxury that industry can't indulge in. Some approaches can then be adopted in bioinformatics to narrow down the huge data to a small fraction of candidates likely carrying the required bioactivity.

First approach - Peptides in common between hydrolysates performing similar functions can identify the key players in each hydrolysate:

We are in the situation where many hydrolysates have been tested, some of which present a positive action that is sought after. For example some hydrolysates may have been tested for their effect on cholesterol and shown to lower cholesterol. The question then becomes, which peptides are the most likely carriers of this functionality? If a number of hydrolysates have been found to perform similar actions on a given disease, their massspectrometry data (consisting of the lists of peptide sequences that are found in these hydrolysates) can be searched for sequences that are common between all the hydrolysates. It is indeed the common segments that are the most likely carriers of this bioactivity. This step will help reduce the number of candidate peptides to a smaller fraction.

Two likely scenarios can occur. Firstly, the list of candidate peptides found using this approach is small enough to synthesize and test each sequence individually in order to expose the true bioactive peptide(s). Secondly, the list of peptides is too large to realistically synthesize and test all of them. In this situation a further computational approach is needed to segregate the different peptides, and to yield an even smaller set. This is where the integration of different bioinformatic methods, some of which are illustrated above, will be useful in reducing the data.

Second approach - Integration of different bioinformatic methods to find novel bioactive peptides:

This second approach is used to further reduce the set of candidate peptides found using the 1st approach. The overall strategy of detecting new bioactive elements cannot succeed without further understanding the characteristics of what one is looking for. One cannot search a hydrolysate aimlessly without a goal. The in-silico prediction of peptides should run in parallel to any hydrolysate testing and mining. 


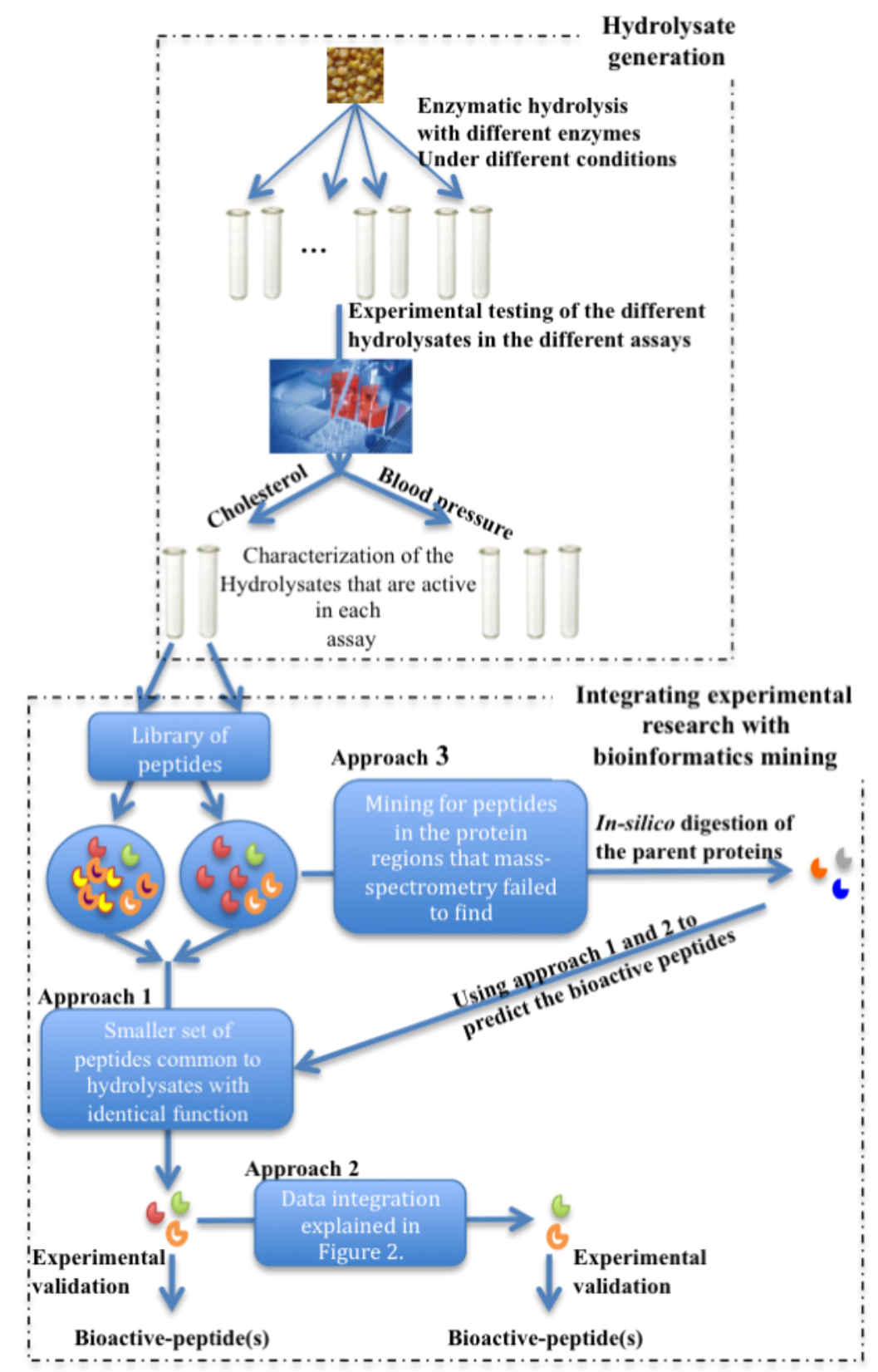

Figure 3. Integrating experimental results with bioinformatics to accelerate bioactive detection in food.

As discussed above, there are many methods that can be integrated together to yield a better predictive power for bioactivity. Some of these were discussed above, these include the evolutionary approach, the amino acid composition preference approach, the mimicking and competition approach. Also as discussed above, some methods may have a higher predictive power on certain disease. This is why it is important to integrate all of these to extract the most information. It is in this second approach where the need for a worldwide functional peptide database is highlighted. Indeed the candidates from the first approach can be used as a query against this database, which will perform all the methods discussed above (together with future methods) and will provide information on how likely each peptide is to be bioactive in a given assay. This of course can only be provided when sufficient information is collected in the database. The more information we find in the literature the better our 
predictive power becomes.

So far these approaches only deal with peptides visible in mass-spectrometry. In many cases the peptides found by mass-spectrometry only cover very little of the parent proteins they derive from. It is not unreasonable to suggest that much of the bioactivity may be in regions that are not seen in mass-spectrometry and these regions should also be searched.

Third approach - Filling the gaps of mass-spectrometry can allow the identification of new bioactive peptides that otherwise would not be identified

This third approach is also computationally oriented (Figure 3). The initial aim of this approach is filling the gaps of mass-spectrometry. Although mass-spectrometry has provided a great source of information of the fragments present in the hydrolysate, it is often limited. Searching computationally for bioactive fragments in some hydrolysates with poor coverage is limited. A way to tackle this issue is by computationally digesting the proteins of the initial food of interest, corn proteins for example. Knowing the enzymes that have been used to generate the hydrolysate one can computationally predict where these enzymes will cleave in the areas that Mass-spectrometry failed to see. Bear in mind that prediction of enzyme digestion can be tricky, especially in regions of post-translational modification. An enzyme may be predicted to cleave a residue, but in reality this residue is glycosylated, in which case the sugars may prevent the enzyme from cleaving at that position. Nonetheless this approach is currently the best way to predict bioactivity in the hidden regions of mass-spectrometry.

The peptides found in this approach can be put through approach 1 and 2 to get a final small set of candidate peptides ready for experimental validation.

\section{CONCLUSION:}

The integration of bioinformatics with food mining has so far been slow. Many issues are hampering the advancement in this field. Some of these include absence of bioinformatic mining tools specifically coded for the food research needs; the insufficient systemization of experimental assay testing of bioactive peptides; a rich central functional food database that bioinformatics can mine.

I described a few successful methods that have found novel bioactive peptides (Figure 1). These methods have been used on a selection of different questions, and have great potential in discovering bioactive peptide in the functional food area (Figure 1).

The diversity of the methods indicates that multiple approaches are needed to find bioactive peptides. Some methods work well for a given disease, while they do not on others. A combination of different bioinformatic methods is needed to mine food for bioactive peptides (Figure 1).

Finally, integrating both bioinformatic and experimental biology is the only way that saves both money and time in discovering new bioactive peptides (Figure 3). I discuss a process that I think will achieve a great yield in mining for new bioactive peptides I food (Figure 3). For this reason, a centralized database accessible by all food laboratories, and containing published and unpublished data is necessary to increase the predictive power of bioinformatics in mining food (Figure 2). This database will implement a variety of approaches found to have been successful in mining for bioactive peptides, as the ones mentioned in the text and shown in Figure 1. 
Acknowledgements: This work was supported by the Irish Research Council for Science, Engineering and Technology, co-funded by Marie Curie Actions under FP7.

Competing Interest: The author declares that she has no competing interest.

\section{REFERENCES:}

1. Paul Ross R, Morgan S, Hill C: Preservation and fermentation: past, present and future. International Journal of Food Microbiology 2002, 79(1-2):3-16.

2. Lee JE, Bae IY, Lee HG, Yang CB: Tyr-Pro-Lys, an angiotensin I-converting enzyme inhibitory peptide derived from broccoli (Brassica oleracea Italica). Food Chem 2006, 99:143-148.

3. Lee NY, Cheng JT, Enomoto T, Nakano Y: One peptide derived from hen ovotransferrin as pro-drug to inhibit angiotensin converting enzyme. J Food Drug Anal 2006, 14:31-35.

4. Miguel M, Aleixandre A: Antihypertensive peptides derived from egg proteins. The Journal of nutrition 2006, 136(6):1457-1460.

5. Mizushima S, Ohshige K, Watanabe J, Kimura M, Kadowaki T, Nakamura Y, Tochikubo O, Ueshima H: Randomized controlled trial of sour milk on blood pressure in borderline hypertensive men. American journal of hypertension 2004, 17(8):701706.

6. Vercruysse L, Van Camp J, Smagghe G: ACE inhibitory peptides derived from enzymatic hydrolysates of animal muscle protein: a review. J Agric Food Chem 2005, 53(21):8106-8115.

7. Motoi H, Kodama $\mathrm{T}$ : Isolation and characterization of angiotensin I-converting enzyme inhibitory peptides from wheat gliadin hydrolysate. Die Nahrung 2003, 47(5):354-358.

8. Kodera $\mathrm{T}$, Nio $\mathrm{N}$ : Identification of an angiotensin I-converting enzyme inhibitory peptides from protein hydrolysates by a soybean protease and the antihypertensive effects of hydrolysates in spontaneously hypertensive model rats. J Food Sci 2006, 71:C164-C173.

9. Nagai T, Suzuki N, Nagashima T: Antioxidative activities and angiotensin Iconverting enzyme inhibitory activities of enzymatic hydrolysates from commercially available Kamaboko species. Food Sci Technol 2006, 12:335-346.

10. Hayes M, Ross RP, Fitzgerald GF, Hill C, Stanton C: Casein-derived antimicrobial peptides generated by Lactobacillus acidophilus DPC6026. Applied and environmental microbiology 2006, 72(3):2260-2264.

11. McCann KB, Shiell BJ, Michalski WP, Lee A, Wan J, Roginski H, Coventry MJ: Isolation and characterisation of a novel antibacterial peptide from bovine $\alpha \mathrm{S} 1$-casein. Int Dairy J 2006, 16:316-323.

12. Mine Y, Kovacs-Nolan J: New insights in biologically active proteins and peptides derived from hen egg. Worlds Poult Sci J 2006, 62:87-95.

13. Hartmann R, Meisel H: Food-derived peptides with biological activity: from research to food applications. Current opinion in biotechnology 2007, 18(2):163-169.

14. Wishart DS: Bioinformatics in drug development and assessment. Drug metabolism reviews 2005, 37(2):279-310. 
15. Edwards RJ, Moran N, Devocelle M, Kiernan A, Meade G, Signac W, Foy M, Park SD, Dunne E, Kenny D et al: Bioinformatic discovery of novel bioactive peptides. Nat Chem Biol 2007, 3(2):108-112.

16. Edwards RJ, Shields DC: BADASP: predicting functional specificity in protein families using ancestral sequences. Bioinformatics 2005, 21(22):4190-4191.

17. Wu J, Aluko RE, Nakai S: Structural requirements of Angiotensin I-converting enzyme inhibitory peptides: quantitative structure-activity relationship study of diand tripeptides. J Agric Food Chem 2006, 54(3):732-738.

18. Fotouhi N, Graves B: Small molecule inhibitors of p53/MDM2 interaction. Current topics in medicinal chemistry 2005, 5(2):159-165.

19. Dempsey EC, Newton AC, Mochly-Rosen D, Fields AP, Reyland ME, Insel PA, Messing RO: Protein kinase $\mathrm{C}$ isozymes and the regulation of diverse cell responses. American journal of physiology Lung cellular and molecular physiology 2000, 279(3):L429-438.

20. Battaini F, Mochly-Rosen D: Happy birthday protein kinase C: past, present and future of a superfamily. Pharmacological research : the official journal of the Italian Pharmacological Society 2007, 55(6):461-466.

21. Griner EM, Kazanietz MG: Protein kinase C and other diacylglycerol effectors in cancer. Nature reviews Cancer 2007, 7(4):281-294.

22. Koya D, King GL: Protein kinase C activation and the development of diabetic complications. Diabetes 1998, 47(6):859-866.

23. Sampson SR, Cooper DR: Specific protein kinase C isoforms as transducers and modulators of insulin signaling. Molecular genetics and metabolism 2006, 89(12):32-47.

24. Inagaki K, Hahn HS, Dorn GW, 2nd, Mochly-Rosen D: Additive protection of the ischemic heart ex vivo by combined treatment with delta-protein kinase $\mathrm{C}$ inhibitor and epsilon-protein kinase C activator. Circulation 2003, 108(7):869-875.

25. Budas GR, Churchill EN, Mochly-Rosen D: Cardioprotective mechanisms of PKC isozyme-selective activators and inhibitors in the treatment of ischemia-reperfusion injury. Pharmacological research : the official journal of the Italian Pharmacological Society 2007, 55(6):523-536.

26. Bright R, Mochly-Rosen D: The role of protein kinase C in cerebral ischemic and reperfusion injury. Stroke; a journal of cerebral circulation 2005, 36(12):2781-2790.

27. Chou WH, Messing RO: Protein kinase $\mathrm{C}$ isozymes in stroke. Trends in cardiovascular medicine 2005, 15(2):47-51.

28. Bowling N, Walsh RA, Song G, Estridge T, Sandusky GE, Fouts RL, Mintze K, Pickard T, Roden R, Bristow MR et al: Increased protein kinase $\mathrm{C}$ activity and expression of Ca2+-sensitive isoforms in the failing human heart. Circulation 1999, 99(3):384-391.

29. Teicher BA: Protein kinase $\mathrm{C}$ as a therapeutic target. Clinical cancer research : an official journal of the American Association for Cancer Research 2006, 12(18):53365345.

30. Aitken A, Ellis CA, Harris A, Sellers LA, Toker A: Kinase and neurotransmitters. Nature 1990, 344(6267):594.

31. Mochly-Rosen D, Khaner H, Lopez J: Identification of intracellular receptor proteins for activated protein kinase C. Proceedings of the National Academy of Sciences of 
the United States of America 1991, 88(9):3997-4000.

32. Budas GR, Koyanagi T, Churchill EN, Mochly-Rosen D: Competitive inhibitors and allosteric activators of protein kinase $\mathrm{C}$ isoenzymes: a personal account and progress report on transferring academic discoveries to the clinic. Biochemical Society transactions 2007, 35(Pt 5):1021-1026.

33. Qvit N, Reuveni H, Gazal S, Zundelevich A, Blum G, Niv MY, Feldstein A, Meushar S, Shalev DE, Friedler A et al: Synthesis of a novel macrocyclic library: discovery of an IGF-1R inhibitor. Journal of combinatorial chemistry 2008, 10(2):256-266.

34. Ye G, Tiwari R, Parang K: Development of Src tyrosine kinase substrate binding site inhibitors. Curr Opin Investig Drugs 2008, 9(6):605-613.

35. Litman P, Ohne O, Ben-Yaakov S, Shemesh-Darvish L, Yechezkel T, Salitra Y, Rubnov S, Cohen I, Senderowitz H, Kidron D et al: A novel substrate mimetic inhibitor of $\mathrm{PKB} / \mathrm{Akt}$ inhibits prostate cancer tumor growth in mice by blocking the PKB pathway. Biochemistry 2007, 46(16):4716-4724.

36. Bogoyevitch MA, Fairlie DP: A new paradigm for protein kinase inhibition: blocking phosphorylation without directly targeting ATP binding. Drug discovery today 2007, 12(15-16):622-633.

37. Michael S, Trave G, Ramu C, Chica C, Gibson TJ: Discovery of candidate KEN-box motifs using cell cycle keyword enrichment combined with native disorder prediction and motif conservation. Bioinformatics 2008, 24(4):453-457.

38. Montanari F, Shields DC, Khaldi N: Differences in the number of disordered regions between yeast duplicated proteins, and their relationship with functional divergence. PLoS One 2011.

39. Rue K, Shields DC, Khaldi N: Co-evolution of disorder in S. cerevisiae interacting proteins. BioSystems 2011.

40. Khaldi N, Shields DC: Shift in the isoelectric-point of milk proteins as a consequence of adaptive divergence between the milks of mammalian species. Biol Direct 2011.

41. Chiba H, Tani F, Yoshikawa M: Opioid antagonist peptides derived from kappacasein. J Dairy Res 1989, 56(3):363-366.

42. Khaldi N, Shields DC: Shift in the isoelectric-point of milk proteins as a consequence of adaptive divergence between the milks of mammalian species. Biol Direct 2011, 6:40.

43. Chabance B, Jolles P, Izquierdo C, Mazoyer E, Francoual C, Drouet L, Fiat AM: Characterization of an antithrombotic peptide from kappa-casein in newborn plasma after milk ingestion. Br J Nutr 1995, 73(4):583-590.

44. Montanari F, Shields DC, Khaldi N: Differences in the number of intrinsically disordered regions between yeast duplicated proteins, and their relationship with functional divergence. PLoS One 2011, 6(9):e24989. 